\title{
Antcin A, a steroid-like compound from Antrodia camphorata, exerts anti-inflammatory effect via mimicking glucocorticoids
}

\author{
Yi-ching $\mathrm{CHEN}^{1}$, Ya-lin LIU², Feng-yin $\mathrm{LI}^{2}$, Chi-I CHANG ${ }^{3}$, Sheng-yang WANG ${ }^{4}$, Kuo-yang LEE ${ }^{5}$, Shun-lai LI ${ }^{6}$, Yi-peng $\mathrm{CHEN}^{6}$,
} Tzyy-rong JINN ${ }^{7, *}$, Jason TC TZEN ${ }^{1,7,8, *}$

${ }^{1}$ Graduate Institute of Biotechnology, ${ }^{2}$ Department of Chemistry and ${ }^{4}$ Department of Forestry, National Chung Hsing University, Taichung 40227, Taiwan, China; ${ }^{3}$ Graduate Institute of Biotechnology, National Pingtung University of Science and Technology, Pingtung 91201, Taiwan, China; ${ }^{5}$ Center for General Education, Southern Taiwan University of Technology, Tainan 71005, Taiwan, China; ${ }^{6}$ Department of Biotechnology, Southern Taiwan University of Technology, Tainan 71005, Taiwan, China; ${ }^{7}$ School of Chinese Medicine, China Medical University, Taichung 40402, Taiwan, China; ${ }^{8}$ Agricultural Biotechnology Research Center, Academia Sinica, Taipei 11529, Taiwan, China

Aim: To determine the active ingredient of Niuchangchih (Antrodia camphorata) responsible for its anti-inflammatory effects and the relevant molecular mechanisms.

Methods: Five major antcins (A, B, C, H, and K) were isolated from fruiting bodies of Niuchangchih. Structural similarity between the antcins and 2 glucocorticoids (cortisone and dexamethasone) was compared. After incubation with each compound, the cytosolic glucocorticoid receptor (GR) was examined for its migration into the nucleus. Mo lecular docking was performed to model the tertiary structure of GR associated with antcins.

Results: Incubation with cortisone, dexamethasone or antcin A (but not antcins B, C, H, and K) led to the migration of glucocorticoid receptor into the nucleus. The minimal concentration of antcin A, cortisone and dexamethasone to induce nuclear migration of glucocorticoid receptor was 10,1, and $0.1 \mathrm{~mol} / \mathrm{L}$, respectively. The results are in agreement with the simulated binding affinity scores of these three ligands docking to the glucocorticoid receptor. Molecular modeling indicates that C-7 of antcin A or glucocorticoids is exposed to a hydrophobic region in the binding cavity of the glucocorticoid receptor, and the attachment of a hydrophilic group to $\mathrm{C}$-7 of the other four antcins presumably results in their being expelled when docking to the cavity.

Conclusion: The anti-inflammatory effect of Niuchangchih is, at least, partly attributed to antcin A that mimics glucocorticoids and triggers translocation of glucocorticoid receptor into nucleus to initiate the suppressing inflammation.

Keywords: antcin A; Antrodia camphorata; cortisone; dexamethasone; glucocorticoid receptor; anti-inflammation

Acta Pharmacologica Sinica (2011) 32: 904-911; doi: 10.1038/aps.2011.36; published online 23 May 2011

\section{Introduction}

Niuchangchih (Antrodia camphorata), growing restrictedly on the inner cavity of the endemic species Cinnamomum kanehirae (Bull camphor tree) Hayata (Lauraceae), is a unique medicinal mushroom found in Taiwan ${ }^{[1]}$. Being a local species, Niuchangchih was originally consumed by the aborigines as a traditional prescription for the discomforts caused by alcohol drinking or exhaustion ${ }^{[2]}$. In the past few decades, fruiting bodies of Niuchangchih have been frequently recom-

\footnotetext{
* To whom correspondence should be addressed.

E-mail jinn@mail.cmu.edu.tw (Tzyy-rong JINN);

TCTZEN@dragon.nchu.edu.tw (Jason TC TZEN)

Received 2010-12-22 Accepted 2011-03-30
}

mended as a folk medicine for the treatment of liver diseases, drug intoxication, diarrhea, abdominal pain, hypertension, and tumorigenic diseases ${ }^{[3]}$. Among the therapeutic effects of Niuchangchih proposed on the basis of practical utilization and scientific investigation, anti-inflammatory effect has been positively highlighted and well-recognized by the consumers, particularly those who suffer in liver dysfunction ${ }^{[4-7]}$.

The compounds identified in Niuchangchih are predominantly benzenoids, diterpenes, triterpenoids, steroids, polysaccharides, and maleic/succinic acid derivatives ${ }^{[8]}$. More than 30 steroid-like compounds (triterpenoids and steroids) identified and structurally determined from fruiting bodies of Niuchangchih represent the major constituents and account for approximately $60 \%$ of dry weight in this medicinal 
mushroom $^{[9]}$. Among these steroid-like compounds, antcins, a unique group having ergostane skeletons, are only found in Niuchangchih to date. Anti-inflammatory, anti-insecticidal and cytotoxic activities have been observed in various antcins and their derivatives ${ }^{[10-16]}$.

Glucocorticoids are a class of steroid hormones that predominantly affect the metabolism of carbohydrates ${ }^{[17]}$. They also play roles in the feedback mechanism of the immune system to repress immune activity (inflammation), and thus are used as the most important anti-inflammatory drugs as well as medicines to treat diseases caused by an overactive immune system ${ }^{[18,19]}$. Glucocorticoids, because of their lipophilic nature, readily diffuse into the cells and initiate antiinflammatory effects by binding to the cytosolic glucocorticoid receptor (GR). The activated glucocorticoid/GR complex migrates into the nucleus where it suppresses the expression of pro-inflammatory proteins and enhances the expression of anti-inflammatory proteins ${ }^{[20]}$.

In light of the consentaneous utilization of glucocorticoids and Niuchangchih in anti-inflammation, we wondered if any constituents in this folk medicine might possess antiinflammatory effects via the same molecular mechanism triggered by glucocorticoids. To inspect this possibility, structural similarity between two glucocorticoids (cortisone and dexamethasone) and five major antcins isolated from fruiting bodies of Niuchangchih was compared. Furthermore, translocation of GR from cytosol to the nucleus after incubation with glucocorticoids or antcins was analyzed. Molecular docking of glucocorticoids and antcins to the binding cavity of GR was simulated and compared.

\section{Materials and methods}

\section{Chemicals and reagents}

Paraformaldehyde, bovine serum albumin (BSA), dimethyl sulfoxide (DMSO), cortisone, and dexamethasone were purchased from Sigma-Aldrich (St Louis, MO, USA). Acetonitrile and phosphoric acid were supplied from Merck (Darmstadt, Germany). F-12 kaighn's modification medium, fetal bovine serum (FBS), Triton X-100, ganoderic acid A, and glycyrrhetic acid were purchased from Hyclone (Logan, UT, USA), Biological Industries (Israel), Amresco (St Louis, MO, USA), Pulin Biotech (Taipei, Taiwan, China), Extrasynthese (Genay, France), respectively.

\section{Chromatography conditions}

Niuchangchih of $3 \mathrm{~g}$ was extracted with methanol $(5 \times 20 \mathrm{~mL})$, and the methanol extract was evaporated to a final volume of $10 \mathrm{~mL}$. The sample was filtered through a $0.45 \mu \mathrm{m}$ filter and analyzed on a liquid chromatography system coupled to a Model 600E photodiode array detector (Waters Corporation, Milford, MA, USA). Chromatographic separations were performed on a Zorbax SB-C18 column $(4.6 \mathrm{~mm} \times 250 \mathrm{~mm}, 5$ $\mu \mathrm{m}$; Agilent, USA) with a guard column, MetaGuard Pursuit C18 (Varian, USA). The mobile phase consisted of solvent A (acetonitrile) and solvent B (0.1\% phosphoric acid). A gradient procedure was used as follows: $23.5 \%$ to $30 \%$ of A for $30 \mathrm{~min}$;
$30 \%$ to $37 \%$ of $\mathrm{A}$ for $90 \mathrm{~min} ; 37 \%$ to $37.3 \%$ of A for $108 \mathrm{~min}$; $37.3 \%$ to $38 \%$ of A for $10 \mathrm{~min}$; $38 \%$ to $47 \%$ of A for $100 \mathrm{~min}$; $47 \%$ to $48.5 \%$ of A for $1 \mathrm{~min} ; 48.5 \%$ to $85 \%$ of A for $95 \mathrm{~min}$; $85 \%$ to $23.5 \%$ of $\mathrm{A}$ for $1 \mathrm{~min}$. The column temperature was set at room temperature, and the injection volume was $10 \mu \mathrm{L}$. The flow rate was set at $1 \mathrm{~mL} / \mathrm{min}$, and the UV absorbance detection wavelength was set at $254 \mathrm{~nm}$.

\section{Preparation of antcins}

The oven-dried fruiting bodies of Niuchangchih (Antrodia camphorata) $(50 \mathrm{~g})$ were extracted with methanol $(3 \times 2 \mathrm{~L})$ at room temperature $(7 \mathrm{~d} \text { each })^{[21]}$. The combined extract was evaporated under reduced pressure to afford a brown residue, which was suspended in $\mathrm{H}_{2} \mathrm{O}(1 \mathrm{~L})$, and then extracted with EtOAc and $n$-BuOH $(3 \times 1 \mathrm{~L})$ sequentially. The EtOAc fraction (10.8 g) was subjected to silica gel chromatography $(60 \mathrm{~cm} \times 3.5 \mathrm{~cm})$ using a stepwise gradient mixture of $n$-hexane and EtOAc as eluent. Ten fractions were collected as follows: 1 [3000 mL, $n$-hexane], 2 [2000 mL, $n$-hexane-EtOAc (19:1)], 3 [2000 mL, $n$-hexane-EtOAc (9:1)], 4 [2000 mL, $n$-hexaneEtOAc (17:3)], 5 [2000 mL, n-hexane-EtOAc (8:2)], 6 [3000 mL, $n$-hexane-EtOAc (7:3)], 7 [3000 mL, n-hexane-EtOAc (5:5)], 8 [3000 mL, n-hexane-EtOAc (4:6)], 9 [3000 mL, n-hexaneEtOAc (2:8)], and 10 (4000 mL, EtOAc). Fraction 5 further purified through a silica gel column $(2 \mathrm{~cm} \times 45 \mathrm{~cm})$, eluted with $\mathrm{CH}_{2} \mathrm{Cl}_{2}$-EtOAc (30:1 to 0:1) to obtain six fractions (each about $300 \mathrm{~mL}), 5 \mathrm{~A}-5 \mathrm{~F}$. Fraction 5C was applied to semipreparative HPLC eluted with $\mathrm{CH}_{2} \mathrm{Cl}_{2}$-acetone (40:1) to yield antcin A $(5.1 \mathrm{mg})$. Fraction 6 was further chromatographed on a silica gel column $(2 \mathrm{~cm} \times 45 \mathrm{~cm})$, eluted with $\mathrm{CH}_{2} \mathrm{Cl}_{2}-\mathrm{EtOAc}(15: 1$ to $0: 1)$ to resolve into five fractions (each about $350 \mathrm{~mL}$ ), 6A-6E. Fraction $6 \mathrm{~B}$ was subjected to semipreparative HPLC eluted with $\mathrm{CH}_{2} \mathrm{Cl}_{2}$-acetone (20:1) to yield antcin B (9.5 mg). Fraction 6C was also subjected to semipreparative HPLC eluted with $\mathrm{CH}_{2} \mathrm{Cl}_{2}$-acetone (15:1) to yield antcin $\mathrm{C}$ (4.5 mg). Fraction 7 was further chromatographed on a silica gel column (2 $\mathrm{cm} \times 45 \mathrm{~cm}$ ), eluted with $\mathrm{CH}_{2} \mathrm{Cl}_{2}$-methanol (50:1) to afford seven fractions (each about $300 \mathrm{~mL}$ ), 7A-7G. Fraction 7C was subjected to semipreparative $\mathrm{HPLC}$ eluted with $\mathrm{CH}_{2} \mathrm{Cl}_{2}$ acetone (9:1) to yield antcin $\mathrm{H}(6.5 \mathrm{mg})$. Fraction 9 was further purified through a silica gel column $(2 \mathrm{~cm} \times 45 \mathrm{~cm})$, eluted with $\mathrm{CH}_{2} \mathrm{Cl}_{2}-\mathrm{MeOH}(15: 1)$ to obtain six fractions (each about 300 $\mathrm{mL}), 9 \mathrm{~A}-9 \mathrm{~F}$. Fraction 9E was subjected to semipreparative HPLC eluted with $\mathrm{CH}_{2} \mathrm{Cl}_{2}$-i-propanol (9:1) to yield antcin $\mathrm{K}$ (4.1 mg). The five purified antcins in a mixture of two epimers at C-25 were confirmed by their spectra of ${ }^{1} \mathrm{H}-\mathrm{NMR},{ }^{13} \mathrm{C}-\mathrm{NMR}$ and HR-EI-MS

\section{General experimental procedures}

NMR spectra were recorded in $\mathrm{CDCl}_{3}$ or $\mathrm{C}_{5} \mathrm{D}_{5} \mathrm{~N}$ at room temperature on a Varian Mercury plus 400 NMR spectrometer, and the solvent resonance was used as internal shift reference (TMS as standard). Thin layer chromatograph was carried out on aluminum-backed silica gel $60 \mathrm{~F}_{254}$ plates (Merck). Silica gel (230-400 mesh ASTM, Merck) was used for column chromatography. 
Detection of GR translocation to the nucleus after drug treatment Human lung cancer cell A549 was obtained from the Bioresources Collection and Research Center, Taiwan, China. Cells were routinely cultured in the F-12k medium supplemented with $10 \%$ FBS and maintained at $37{ }^{\circ} \mathrm{C}$ in a humidified atmosphere with $5 \% \mathrm{CO}_{2}$. They were cultured on glass coverslips in $6 \mathrm{~cm}$ dishes at a density of $2 \times 10^{5}$ cells for $24 \mathrm{~h}$, serum-starved for $6 \mathrm{~h}$, and then treated with each of the test compounds (dissolved in DMSO) for $1 \mathrm{~h}$. After treatment, cells were fixed with $4 \%$ paraformaldehyde, permeabilized with $0.5 \%$ Triton-X 100, blocked with 3\% BSA in phosphate buffered saline (PBS) buffer for $30 \mathrm{~min}$, and incubated with rabbit polyclonal anti-GR antibodies (1:200 dilution; Santa Cruz, CA, USA) for $2 \mathrm{~h}$. After antibody incubation, cells were washed with the PBS buffer, incubated with Alexa Fluor 488 anti-rabbit IgG (1:1000 dilution; Molecular Probes, Eugene, OR, USA) for $1 \mathrm{~h}$, and then stained with $0.5 \mu \mathrm{g} / \mathrm{mL}$ of $4^{\prime}$, 6diamidino-2-phenylindole (DAPI) for $1 \mathrm{~min}$ (Molecular Probes, Eugene, OR, USA). Cellular images were visualized in an Olympus IX71 fluorescence microscope system (Olympus, Tokyo, Japan), and photos were taken by a digital camera with UPlanSApo $60 \times / 1.35$ oil immersion objective lens.

\section{Molecular modeling}

The X-ray structure of GR in complex with deacylcortivazol (DAC), a potent glucocorticord, and steroid receptor coactivator-1 (SRC-1) (PDB code: 3BQD) ${ }^{[22]}$ was used as the template for molecular docking. In order to facilitate docking process, we removed DAC and SRC-1 from GR, as well as the surrounding water molecules. Noticing that cortisone was converted to cortisol, a more active form, by $11 \beta$-hydroxysteroid dehydrogenase in liver ${ }^{[23]}$, we assumed that the carbonyl group attached to C-11 of antcin A was possibly converted to a hydroxyl group in the A549 cells by their endogenous $11 \beta$-hydroxysteroid dehydrogenase activity ${ }^{[24]}$. Thus, antcin A (11 $\beta$-hydroxyl form), cortisol and dexamethasone were used as GR ligands for docking simulation, and they were constructed and minimized with consistent force field ${ }^{[25]}$ using the Discover Studio 2.0 (DS) package (http://accelrys.com/ about/legal/ $)^{[26]}$.

The binding cavity for GR ligands was analyzed using DS package and defined as the region of the sphere with a $16 \AA$ radius from the center of the binding cavity, which lies in the area among Leu536, Trp600, and Met646. Docking of antcin A, cortisol and dexamethasone to GR was performed in silico by employing the CDOCKER module ${ }^{[27]}$ in the DS environment. CDOCKER is a grid-based molecular docking method which employs CHARMm forcefield ${ }^{[28]}$ and assigns the partial charges of the atoms with those found in Merck Molecular Force Field (MMFF) ${ }^{[29]}$. For each GR ligand studied, 300 different conformations were generated using high temperature molecular dynamics simulation and five conformations with the lowest energies were selected as the candidates for docking. The high temperature MD simulation offers all the advantages of full ligand flexibility (including bonds, angles and dihedrals) and reasonable computation time. In this study, the high temperature was set to be $1000 \mathrm{~K}$. After these conformations were translated into the binding sphere, the candidate poses were created by using random rigid-body rotations followed by simulated annealing and final energy minimization, while keeping the GR structure fixed during all the above processes except the annealing. The ligand pose with the highest CDOCKER energy was selected to represent each of the studied GR ligands inside the binding cavity, and employed MMFF forcefield to minimize the final structure. In order to validate our approach, the redocking of DAC onto GR was performed and the RMSD value of the redocked DAC/ GR complex compared with the X-ray one was $0.92 \AA$.

For the evaluation of the binding affinity, the scoring method piecewise linear potential (PLP) ${ }^{[30]}$ implemented in DS was applied. PLP was reported to give reliable correlations between the binding scores and experimentally determined protein-ligand binding affinities as reported previously ${ }^{[31]}$. Two type of PLP functions are available, PLP1 and PLP2. In this study, PLP2 was adopted since it includes an extra set of parameters to describe atomic size effect, which is absent in PLP1. In this study, a hydrogen bond (X-H...Y) involves $X$ and $Y$ being two different electronegative atoms, such as oxygen, nitrogen and fluorine, with the distance between $\mathrm{H}$ and $\mathrm{Y}$ ranging from 1.2 up to $2.76 \AA$ and angle of $\mathrm{X}-\mathrm{H}-\mathrm{Y}$ between 120 and 180 degrees.

\section{Results}

Purification of major antcins from fruiting bodies of Niuchangchih Five major antcins (A, B, C, H, and K) were identified in the methanol extract of fruiting bodies of Niuchangchih, and two C-25 epimers were found in each of the five antcins (Figure 1). The five antcins were separately purified as a mixture of two C-25 epimers, and the correctness of these compounds was confirmed by comparing their spectral data of NMR and Mass (data not shown) with those of antcins described in the literatures $^{[13,21,32]}$.

\section{Structural comparison between antcins and glucocorticoids}

Structurally similar to cortisone (the major natural glucocorticoid) and dexamethasone (a synthetic glucocorticoid with

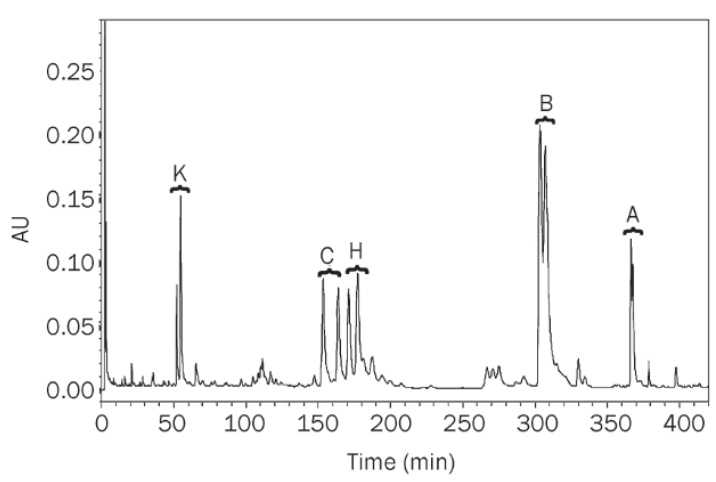

Figure 1. HPLC profile (0-420 $\mathrm{min}$ ) of the methanol extract of Niuchangchih at $254 \mathrm{~nm}$. Positions of five major antcins (A, B, C, H, and K) are indicated on top of the peaks. Two epimers are found in each antcin. 
activity stronger than cortisone), antcins comprise a core steroidal backbone with carbonyl or hydroxyl groups attached to C-3 and C-11; and yet, the head groups attached to C-17 of glucocorticoids and antcins are different (Figure 2). Having a carbonyl group at C-11, cortisone is more similar to antcins than dexamethasone that possesses a hydroxyl group at C-11 and a fluoro-replacement at C-9. Among the five antcins, antcin $\mathrm{A}$ is more similar to cortisone than the other four antcins which have an additional carbonyl or hydroxyl group attached to C-7.

\section{Nuclear migration of GR treated with antcins}

By using two positive controls (cortisone and dexamethasone) and two negative controls (ganoderic acid A and glycyrrhetic acid), induction of GR translocation from cytosol to the nucleus was measured to evaluate if antcins could lead to a similar anti-inflammatory effect via the same mechanism triggered by glucocorticoids. The results showed that antcin A, similar to the two positive controls, led to nuclear migration of GR as the green fluorescence (indicating the localization of GR) was exclusively confined in the nuclei that were also visualized by blue fluorescence stained with DAPI (Figure 3). In contrast, green fluorescence was found diffusive in the whole cells (larger than the blue fluorescence stained with DAPI) when treated with the other four antcins or the two negative controls. To inspect the relative capability of these ligands for activating GR, three concentrations, $0.1,1$, and $10 \mu \mathrm{mol} / \mathrm{L}$ of antcin $\mathrm{A}$, cortisone and dexamethasone were examined in the same test. The results showed that antcin $A$, cortisone and dexamethasone led to nuclear migration of GR at 10,1, and 0.1 $\mu \mathrm{mol} / \mathrm{L}$, respectively (Figure 4). Thus, dexamethasone pos- sessed the highest capability while antcin A had the lowest capability for activating GR. Relatively, the minimal concentration of antcin A required for nuclear migration of GR was approximately 10 times higher than that of cortisone.

\section{Molecular modeling and docking of antcin A to GR}

Molecular modeling showed that antcin A (11 $\beta$-hydroxyl form) could successfully dock to the binding cavity of GR (Figure $5 \mathrm{~A}$ ). In comparison with the docking of dexamethasone and cortisol (11 $\beta$-hydroxyl form converted from cortisone by $11 \beta$-hydroxysteroid dehydrogenase in liver), antcin A was found located in the same binding cavity of GR with similar orientation and interaction since these three GR ligands could be superimposed correspondingly (Figure 5B). Detailed comparison indicates that Gln642, Met646, and Tyr735 are slightly dislocated in antcin A/GR complex when compared with those in the other two complexes, and that Arg611 forming a hydrogen bond with cortisol or dexamethasone is missing in antcin A/GR. The distance between R611 and antcin A is 3.06 $\AA$. However, for dexamethasone and cortisol, the distances are 1.62 and $1.52 \AA$, respectively. Consensually observed in the docking of antcin A, cortisol and dexamethasone to GR, the hydrophobic side of the steroidal backbone where C-7 is located is surrounded by hydrophobic residues in the binding cavity, including Leu608, Met604, Phe623, Ala605, Met601, Met646, Leu732, and Tyr735 (Figure 5C). Moreover, theoretical calculation showed that the binding affinity scores (PLP2 values) of antcin $A$, cortisol and dexamethasone to GR were -95.0, -96.4, and -104.1, respectively. These data are in agreement with the results observed in their minimal concentrations required for nuclear migration of GR (Figure 4).
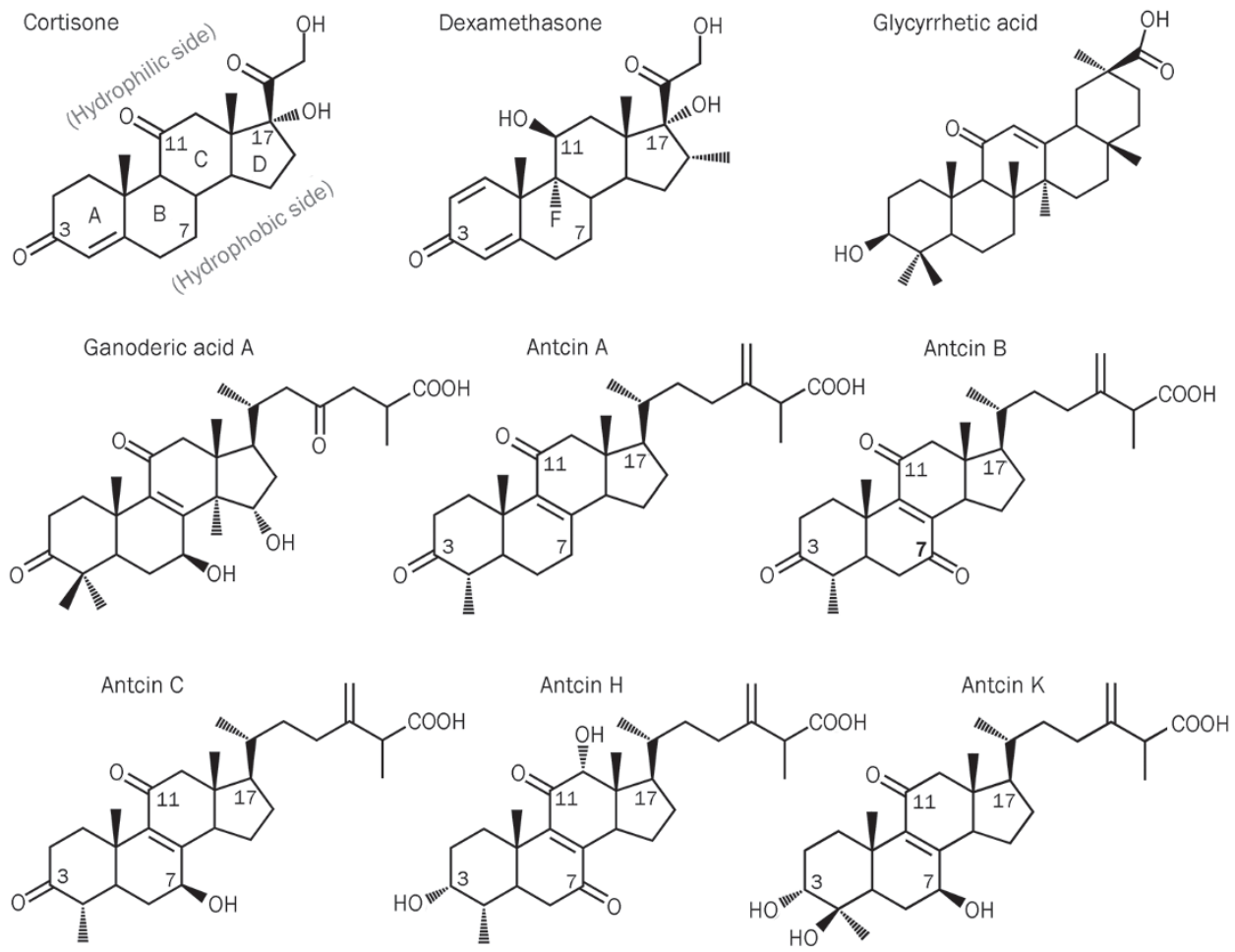

Figure 2. Chemical structures of cortisone, dexamethasone, glycyrrhetic acid, ganoderic acid $\mathrm{A}$, and five major antcins (A, B, C, H, and K) of Niuchangchih. 


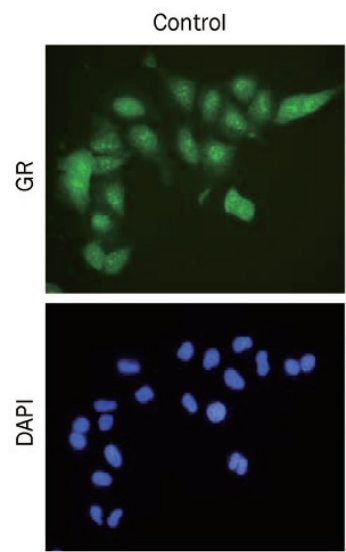

Antcin A
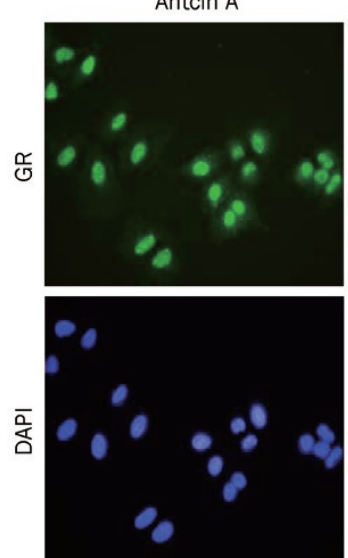
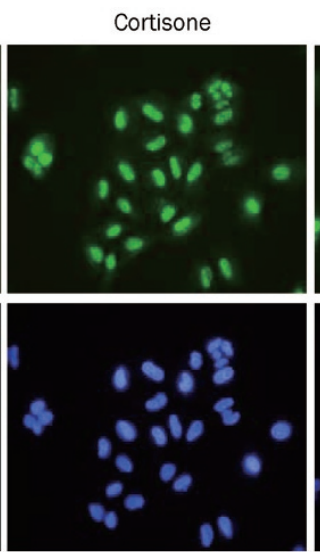

Antcin B
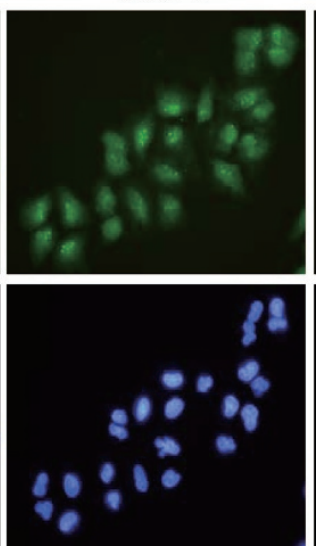

Dexamethasone
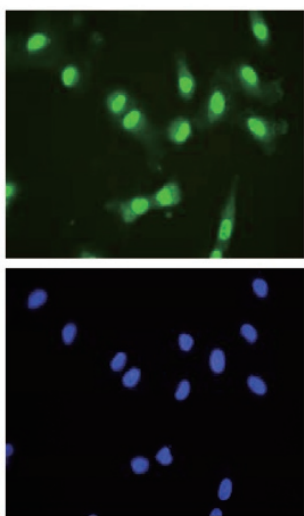

Antcin C
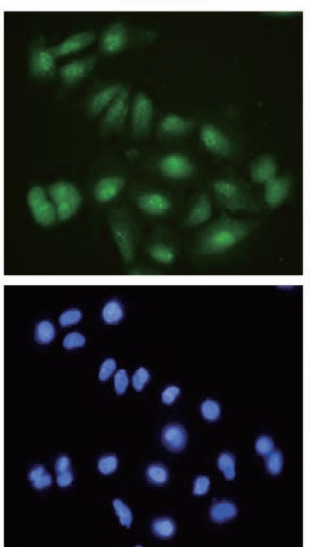

Glycyrrhetic acid
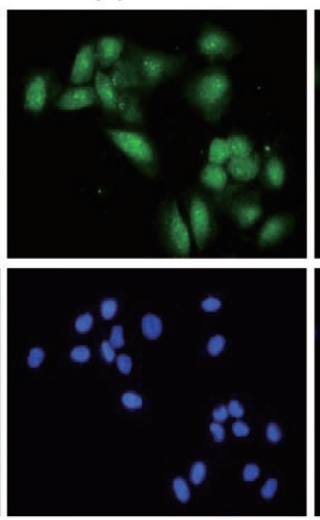

Antcin $\mathrm{H}$
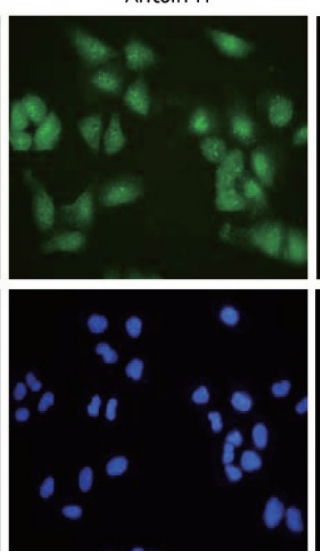

Ganoderic acid A

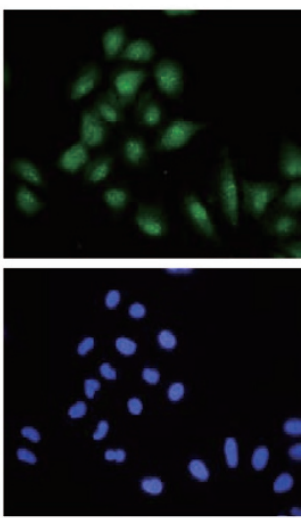

Antcin K

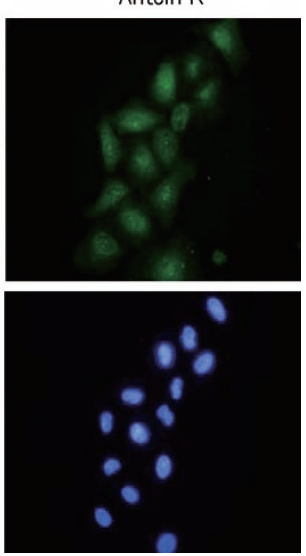

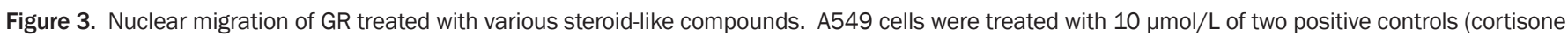

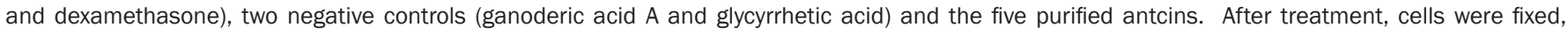

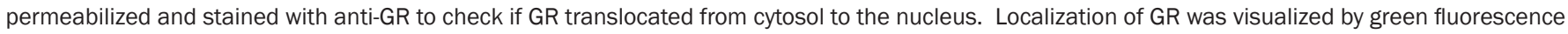
of anti-GR, and nuclei were visualized by blue fluorescence stained with DAPI.

\section{Modeling comparison between antcin A/GR and dexametha- sone/GR}

The validation of our docking results was also verified by examining the docking of dexamethasone to GR reported previously $^{[33,34]}$. Dexamethasone is oriented with its A ring anchored by two hydrogen bonds to the carbonyl inside the binding cavity of GR and with its $\mathrm{D}$ ring hanging around the cavity opening. Six residues of GR, Asn564, Gln570, Arg611, Gln642, Cys736, and Thr739 found on one side of the steroidal backbone are involved in hydrogen bonding with the specific functional groups of dexamethasone in accord with the hydrogen bonding network observed in the crystal of dexamethasone/GR complex (PDB code 1M2Z) ${ }^{[33]}$. Several distinct structural characteristics are present between antcin A and dexamethasone, mainly in their head groups attached to C-17 of the D ring. Firstly, the head group (17.3 $\mathrm{A}$ ) of antcin $\mathrm{A}$ is longer than that $(12.9 \AA)$ of dexamethasone. Furthermore, dexamethasone is neutral in its native form, but antcin $\mathrm{A}$ is ionic at $\mathrm{pH} 7$ due to the carboxylate at the end of its head group. There are three hydrophilic groups in the head group of dexamethasone, but only one in that of antcin A. This explains why only three residues in GR, ie Gln570, Asn564, and Thr739, form hydrogen bonds with antcin A.

Besides the reduction in the number of hydrogen bonds, antcin A suffers two other disadvantages when compared with dexamethasone. First, as the carboxylate in the end of the long head group of antcin A forms a hydrogen bond with Thr739, it is pulled by this hydrogen bond toward the cavity opening. This shift weakens the strength of the hydrogen bonding network and causes a slight mismatch of the hydrophobic interaction pattern. Second, the replacement of the carbonyl group with methyl group in the head group pushes Gln642, Met646, and Tyr735 away from antcin A. The consequence of the dislocation of Gln642, Met646, and Tyr735 is to make the cavity opening slightly larger than that found in the dexamethasone/ GR complex. Taken together, the GR binding affinity of antcin $\mathrm{A}$ is lower than that of dexamethasone in molecular modeling.

\section{Discussion}

In accord with their structural similarity, antcin A extracted from fruiting bodies of Niuchangchih led to GR translocation from cytosol to the nucleus as cortisone did in our experimental conditions. Molecular modeling showed that antcin A as well as glucocorticoids could stably interact with the binding 

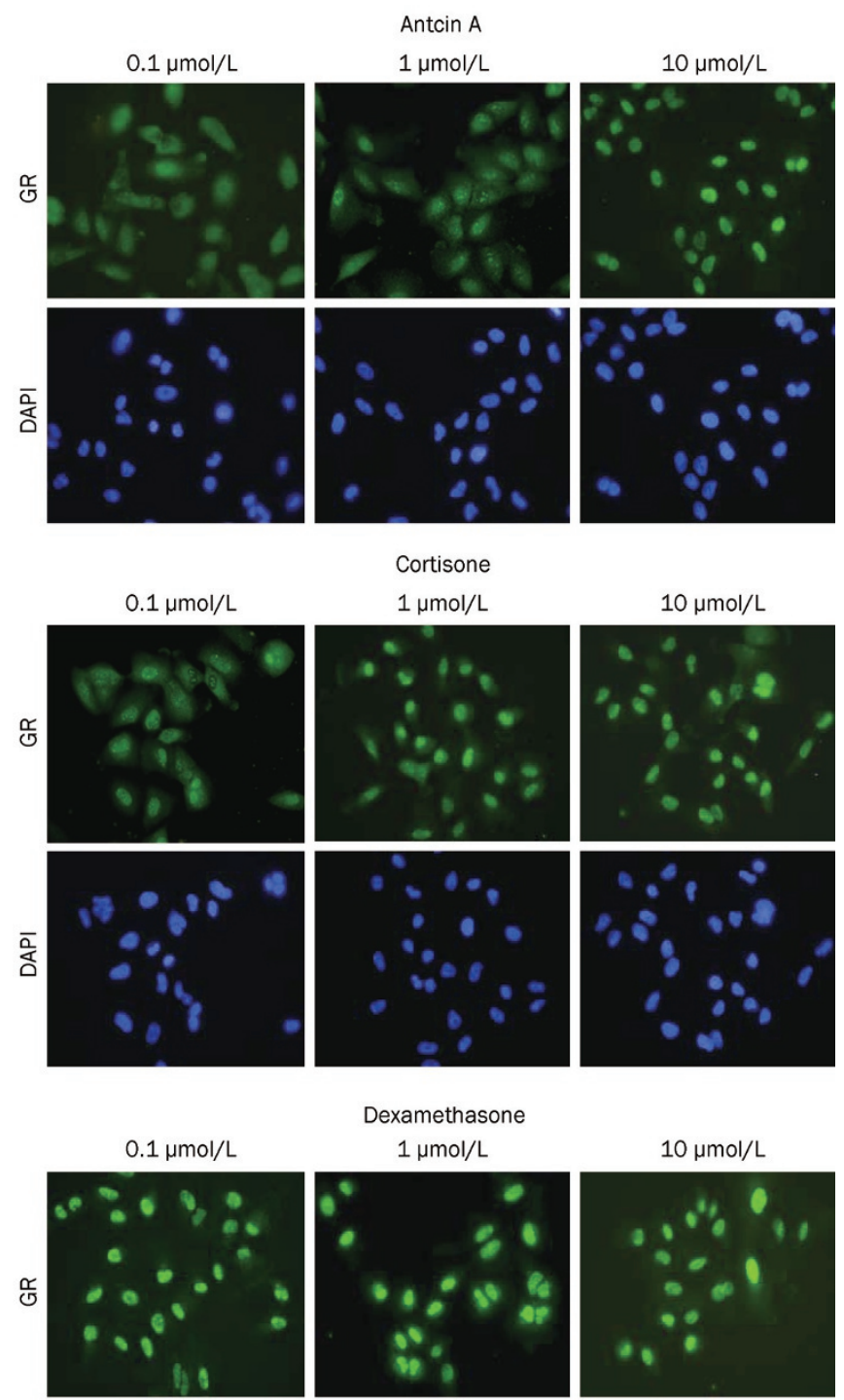

Dexamethasone
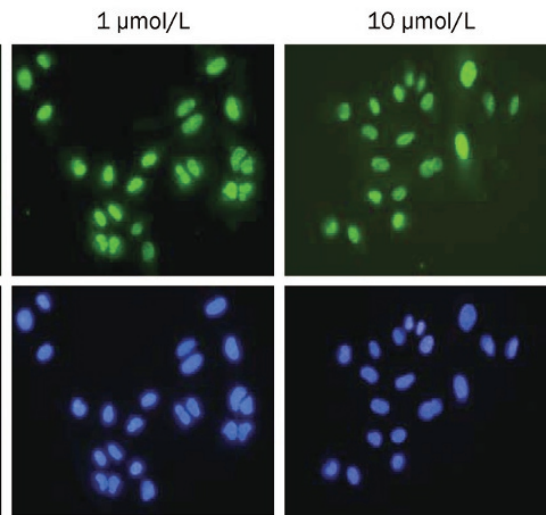

Figure 4. Nuclear migration of GR treated with antcin A, cortisone and dexamethasone at three different concentrations, 0.1, 1, and $10 \mu \mathrm{mol} / \mathrm{L}$. Similar fluorescence imaging in A549 cells was visualized as described in Figure 3.

cavity of GR despite the fact that their head groups at C-17 are different. Taken together, we propose that antcin A may act as an active ingredient responsible for the anti-inflammatory effect of Niuchangchih via the same molecular mechanism triggered by glucocorticoids (Figure 6): mimicking glucocorticoids, antcin A diffuses across the cell membrane and binds to the cytosolic GR that forms a dimer after dissociated from the heat-shock protein and then translocates into the nucleus to initiate the suppression of inflammation at the gene regulation level ${ }^{[20]}$.

In this study, five major antcins were isolated from fruiting bodies of Niuchangchih and examined for their possible therapeutic effects by mimicking glucocorticoids. Surprisingly, only antcin A, but not the remaining four, could lead to nuclear migration of GR. The main distinction between antcin $\mathrm{A}$ and the other four antcins is the additional carbonyl or hydroxyl group attached to C-7 of the latter (Figure 2). Molecular modeling indicates that C-7 of antcin A or glucocorticoids is exposed to a hydrophobic region in the binding cavity of GR (Figure 5C), and thus the other four antcins with the additional hydrophilic group attached to C-7 are presumably expelled when they approach GR. Similarly, repulsion is expected between the hydrophobic region in the binding cavity of GR and the hydrophilic hydroxyl group attached to C-7 of ganoderic acid A (a negative control in Figure 3) that possesses a head group at C-17 very similar to that of antcins. In order to demonstrate the adverse effect caused by the mismatch between the hydrophilic group attached to C-7 of the antcins and their surrounding environments in the docked complexes, the binding affinity score of antcin B was calculated, which is -89.3 , higher than that of antcin A, -95. Regarding the head group, the linear chain attached to antcin A is longer than that attached to glucocorticoids and apparently reduces the binding affinity to GR. Moreover, replacement of the short head group in glucocorticoids with an additional fusion ring, ie the fifth ring of pentacyclic triterpenoids, seems to be unacceptable for the binding to GR as observed in the treatment with glycyrrhetic acid (Figure 3) that comprises a core steroidal backbone very similar to glucocorticoids with a hydroxyl group at $\mathrm{C}-3$ and a carbonyl group at C-11.

To date, a total of 10 antcins, (A, B, C, D, E, F, G, H, I, and $\mathrm{K})$ plus some of their derivatives have been identified in fruiting bodies of Niuchangchih ${ }^{[9]}$. Among the five minor ones not examined in this study, antcins D, F, G, and I, but not antcin $\mathrm{E}$, possess an additional hydrophilic group attached to C-7. Therefore, we speculate that antcin E would be capable of mimicking glucocorticoids, similar to antcin A, to trigger the translocation of GR into nucleus, and thus contribute to a limited or minor extent to the anti-inflammatory effect of Niuchangchih. Furthermore, whether the other eight antcins may act as prodrugs with the additional hydrophilic group at C-7 removed by intestinal microflora or the enterohepatic circulation remains to be investigated.

In the past few decades, medicinal effects of Niuchangchih have been well-recognized by the consumers who suffer in liver dysfunction including liver cancer ${ }^{[8]}$. Recently, antcin A related compounds, particularly its methyl derivative, was demonstrated to induce apoptosis in human liver cancer cells $^{[35]}$. Intracellular reactive oxygen species generation and NADPH oxidase activation were observed in the cells treated with methyl antcin A, and the apoptosis was proposed to be induced through oxidant-mediated cofilin- and Bas-triggered mitochondrial pathway. It will be an interesting task to figure out whether any cross-talk interaction is present between the apoptosis signaling pathway induced by antcin A derivatives 

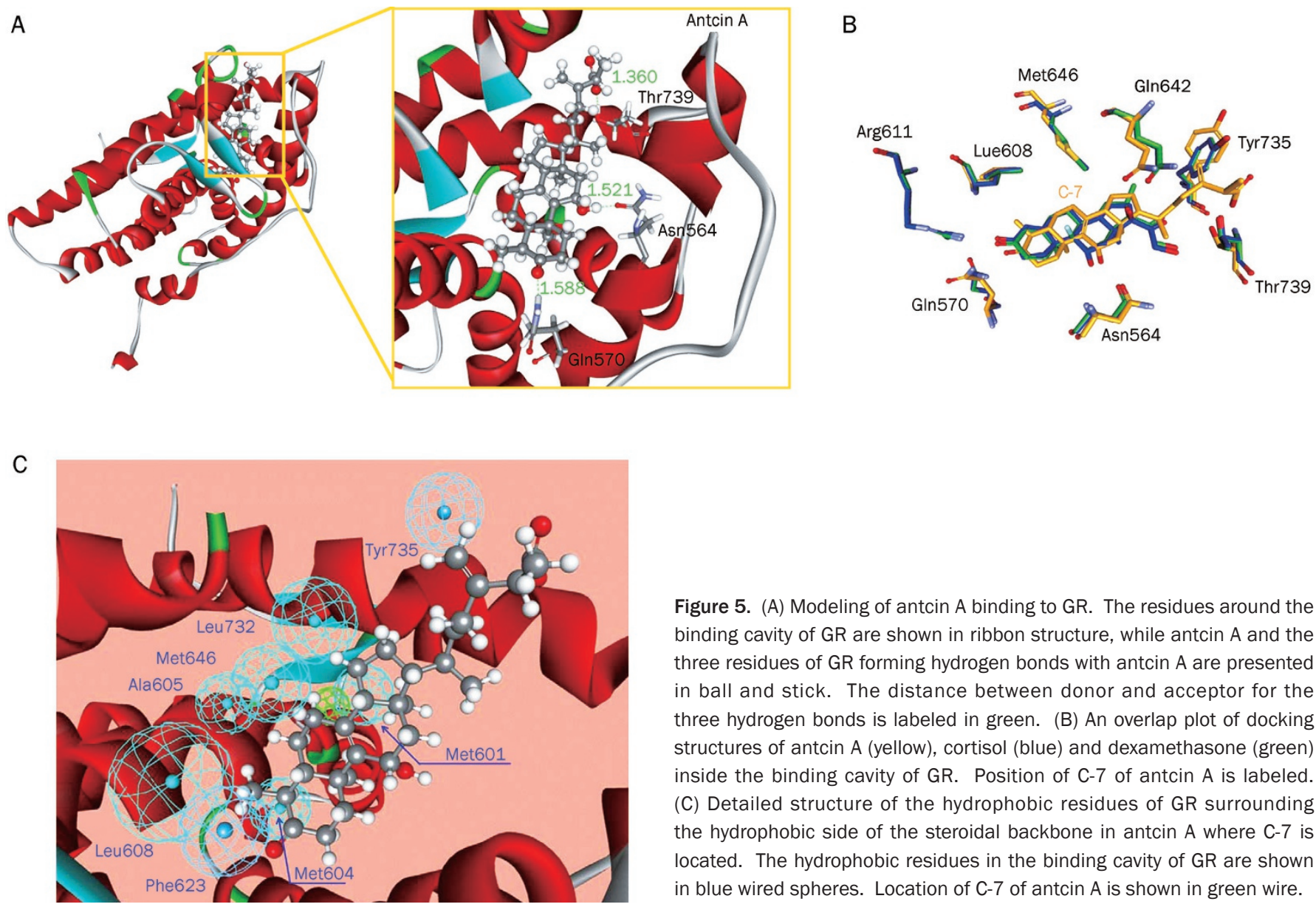

Figure 5. (A) Modeling of antcin A binding to GR. The residues around the binding cavity of GR are shown in ribbon structure, while antcin $A$ and the three residues of GR forming hydrogen bonds with antcin $A$ are presented in ball and stick. The distance between donor and acceptor for the three hydrogen bonds is labeled in green. (B) An overlap plot of docking structures of antcin A (yellow), cortisol (blue) and dexamethasone (green) inside the binding cavity of GR. Position of C-7 of antcin A is labeled. (C) Detailed structure of the hydrophobic residues of GR surrounding the hydrophobic side of the steroidal backbone in antcin A where C-7 is located. The hydrophobic residues in the binding cavity of GR are shown in blue wired spheres. Location of C-7 of antcin A is shown in green wire.

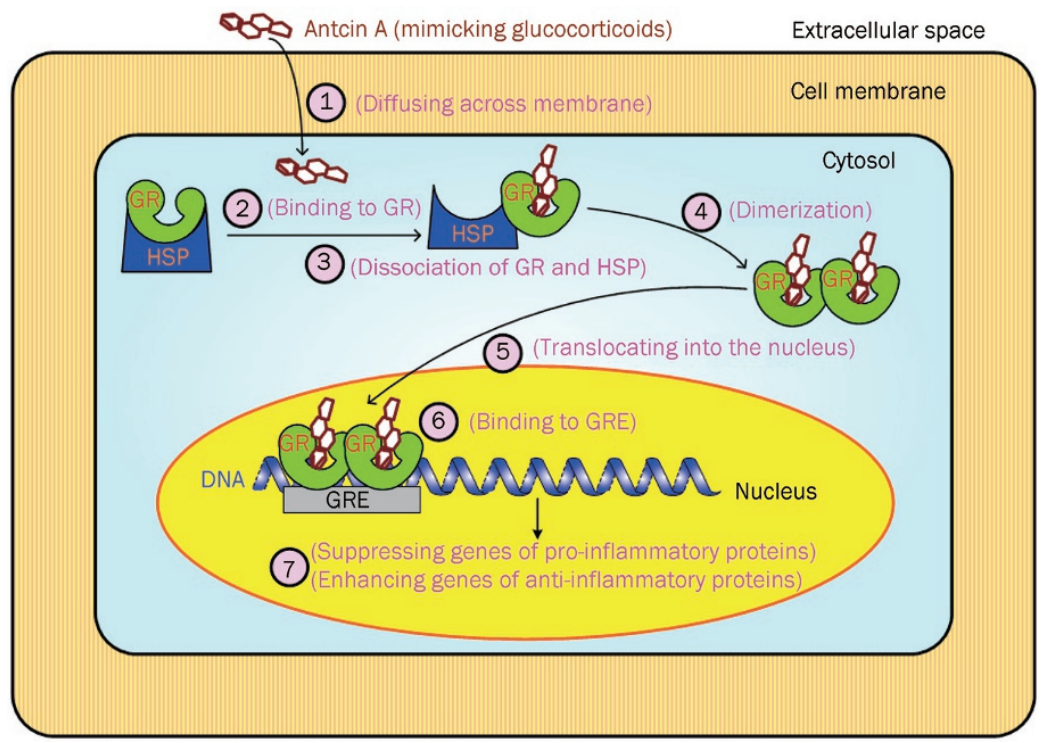

Figure 6. Proposed molecular mechanism responsible for the anti-inflammatory effect of antcin A in Niuchangchih. Step 1: Antcin A diffuses across cell membrane by its lipophilic character. Step 2: Antcin A binds to the cytosolic GR. Step 3: After binding with antcin A, GR dissociates with the heat-shock protein (HSP). Step 4: Dimerization of antcin A/GR complex. Step 5: Antcin A/GR translocates into the nucleus. Step 6: Antcin A/GR binds to glucocorticoid responsive elements (GRE) of target genes. Step 7: Regulation of gene expression, such as suppressing the expression of pro-inflammatory proteins and enhancing the expression of anti-inflammatory proteins, leads to the anti-inflammatory effect.

and the anti-inflammatory signaling pathway induced by antcin A/GR complex, particularly in liver cells.

Although glucocorticoids are effective anti-inflammatory drugs, short-term side effects, such as insomnia, euphoria and, mania as well as long-term side effects, such as Cushing's syn- drome, truncal weight gain, osteoporosis, and glaucoma have been observed ${ }^{[18]}$. The underlying molecular mechanisms for these side effects are complex, distinct and only partly understood; they are presumably mediated via transactivation and/ or transrepression on a case-by-case basis. Searching for new 
drugs that exert the anti-inflammatory effect with no or low side effects is an on-going task. It remains to be investigated whether the utilization of antcin A at therapeutic dosage for anti-inflammation leads to the same or different side effects comparable with those of glucocorticoids.

\section{Acknowledgements}

Project was supported by a grant to Jason TC TZEN from the National Science Council, Taiwan, China (No 98-2622-B-005007-CC3).

\section{Author contribution}

Jason TC TZEN and Tzyy-rong JINN designed research; Yiching CHEN, Ya-lin LIU, Chi-I CHANG, Sheng-yang WANG performed research; Kuo-yang LEE, Shun-lai LI, and Yi-peng CHEN contributed new analytical tools and reagents; Jason TC TZEN and Feng-yin LI wrote the paper.

\section{References}

1 Wu SH, Ryvarden L, Chang TT. Antrodia camphorata ("niu-changchih"), new combination of a medicinal fungus in Taiwan. Bot Bull Acad Sin 1997; 38: 273-5.

2 Su CH. Health Guardian Angel: Antrodia camphorata. 1 st ed. Taipei (China): EKS Book Publishing Co; 2002.

3 Chen CJ, Su CH, Lan MH. Study on solid cultivation and bioactivity of Antrodia camphorata. Fung Sci 2001; 16: 65-72.

4 Hseu YC, Wu FY, Wu JJ, Chen JY, Chang WH, Lu FJ, et al. Antiinflammatory potential of Antrodia Camphorata through inhibition of iNOS, COX-2 and cytokines via the NF-kappaB pathway. Int Immunopharmacol 2005; 5: 1914-25.

5 Shen YC, Wang YH, Chou YC, Chen CF, Lin LC, Chang TT, et al. Evaluation of the anti-inflammatory activity of zhankuic acids isolated from the fruiting bodies of Antrodia camphorata. Planta Med 2004; 70: 310-4.

6 Liu DZ, Liang HJ, Chen $\mathrm{CH}$, Su CH, Lee TH, Huang CT, et al. Comparative anti-inflammatory characterization of wild fruiting body, liquidstate fermentation, and solid-state culture of Taiwanofungus camphoratus in microglia and the mechanism of its action. J Ethnopharmacol 2007; 113: 45-53.

7 Huang $\mathrm{CH}$, Chang YY, Liu CW, Kang WY, Lin YL, Chang HC, et al. Fruiting body of Niuchangchih (Antrodia camphorata) protects livers against chronic alcohol consumption damage. J Agric Food Chem 2010; 58: 3859-66.

8 Ao ZH, Xu ZH, Lu ZM, Xu HY, Zhang XM, Dou WF. Niuchangchih (Antrodia camphorata) and its potential in treating liver diseases. J Ethnopharmacol 2009; 121: 194-212.

9 Geethangili M, Tzeng YM. Review of pharmacological effects of Antrodia camphorata and its bioactive compounds. Evid Based Complement Alternat Med 2011; 2011; 212641. doi:10.1093/ecam/ nep108.

10 Cherng IH, Wu DP, Chiang HC. Triterpenoids from Antrodia cinnamomea. Phytochemistry 1996; 41: 263-67.

11 Shen YC, Chen CF, Wang YH, Chang TT, Chou CJ. Evaluation of the immuno-modulating activity of some active principles isolated from the fruiting bodies of Antrodia camphorata. Chin Pharm J 2003; 55: 313-8.

12 Shen CC, Wang YH, Chang TT, Lin LC, Don MJ, Hou YC, et al. Antiinflammatory ergostanes from the basidiomata of Antrodia salmonea. Planta Med 2007; 73: 1208-13.

13 Chen $\mathrm{CH}$, Yang SW, Shen YC. New steroid acids from Antrodia cinnamomea, a fungal parasite of Cinnamomum micranthum. J Nat Prod 1995; 58: 1655-61.

14 Chen JJ, Lin WJ, Liao CH, Shieh PC. Anti-inflammatory benzenoids from Antrodia camphorata. J Nat Prod 2007; 70: 989-92.
15 Male KB, Rao YK, Tzeng YM, Montes J, Kamen A, Luong JH. Probing inhibitory effects of Antrodia camphorata isolates using insect cellbased impedance spectroscopy: inhibition vs chemical structure. Chem Res Toxicol 2008; 21: 2127-33.

16 Yeh CT, Rao YK, Yao CJ, Yeh CF, Li CH, Chuang SE, et al. Cytotoxic triterpenes from Antrodia camphorata and their mode of action in HT29 human colon cancer cells. Cancer Lett 2009; 285: 73-9.

17 Rose AJ, Vegiopoulos A, Herzig S. Role of glucocorticoids and the glucocorticoid receptor in metabolism: insights from genetic manipulations. J Steroid Biochem Mol Biol 2010; 122: 10-20.

18 De Bosscher K, Haegeman G, Elewaut D. Targeting inflammation using selective glucocorticoid receptor modulators. Curr Opin Pharmacol 2010; 10: 497-504.

19 Schacke H, Docke WD, Asadullah K. Mechanisms involved in the side effects of glucocorticoids. Pharmacol Ther 2002; 96: 23-43.

20 Revollo JR, Cidlowski JA. Mechanisms generating diversity in glucocorticoid receptor signaling. Ann N Y Acad Sci 2009; 1179: 167-78.

21 Cherng IH, Chiang HC, Cheng MC, Wang Y. Three new triterpenoids from Antrodia cinnamomea. J Nat Prod 1995; 58: 365-71.

22 Suino-Powell K, Xu Y, Zhang C, Tao YG, Tolbert WD, Simons SS Jr, et al. Doubling the size of the glucocorticoid receptor ligand binding pocket by deacylcortivazol. Mol Cell Biol 2008; 28: 1915-23.

23 Chapman KE, Coutinho AE, Gray M, Gilmour JS, Savill JS, Seckl JR. The role and regulation of 11 beta-hydroxysteroid dehydrogenase type 1 in the inflammatory response. Mol Cell Endocrinol 2009; 301: 123-31.

24 Sai S, Esteves CL, Kelly V, Michailidou Z, Anderson K, Coll AP, et al. Glucocorticoid regulation of the promoter of 11beta-hydroxysteroid dehydrogenase type 1 is indirect and requires CCAAT/enhancerbinding protein-beta. Mol Endocrinol 2008; 22: 2049-60.

25 Lifson S, Warshel A. Consistent force field for calculations of conformations, vibrational spectra, and enthalpies of cycloalkane and n-alkane molecules. J Chem Phys 1968; 49: 5116-29.

26 Accelrys Software Inc, Discovery studio modeling environment, Release 2.0, San Diego: Accelrys Software Inc, 2007.

27 Wu G, Robertson DH, Brooks CL 3rd, Vieth M. Detailed analysis of grid-based molecular docking: A case study of CDOCKER-A CHARMmbased MD docking algorithm. J Comput Chem 2003; 24: 1549-62.

28 Brooks BR, Bruccoleri RE, Olafson BD, States DJ, Swaminathan S, Karplus M. CHARMM: A program for macromolecular energy minimization and dynamics calculations. J Comp Chem 1983; 4: 187-217.

29 Halgren TA. Merck Molecular Force Field. II. MMFF94 van der Waals and electrostatic parameters for intermolecular interactions. J Comp Chem 1996; 17: 520-52.

30 Gehlhaar DK, Verkhivker GM, Rejto PA, Sherman CJ, Fogel DB, Fogel $\mathrm{LJ}$, et al. Molecular recognition of the inhibitor AG-1343 by HIV-1 protease: conformationally flexible docking by evolutionary programming. Chem Biol 1995; 2: 317-24.

31 Wang R, Lu Y, Wang S. Comparative evaluation of 11 scoring functions for molecular docking. J Med Chem 2003; 46: 2287-303.

32 Shen CC, Kuo YC, Huang RL, Lin LC, Don MJ, Chang TT, et al. New ergostane and lanostane from Antrodia camphorata. J Chin Med 2003; 14: 247-58.

33 Bledsoe RK, Montana VG, Stanley TB, Delves CJ, Apolito CJ, McKee $\mathrm{DD}$, et al. Crystal structure of the glucocorticoid receptor ligand binding domain reveals a novel mode of receptor dimerization and coactivator recognition. Cell 2002; 110: 93-105.

34 Kauppi B, Jakob C, Farnegardh M, Yang J, Ahola H, Alarcon M, et al. The three-dimensional structures of antagonistic and agonistic forms of the glucocorticoid receptor ligand-binding domain: RU-486 induces a transconformation that leads to active antagonism. J Biol Chem 2003; 278: 22748-54.

35 Hsieh YC, Rao YK, Wu CC, Huang CY, Geethangili M, Hsu SL, et al. Methyl antcinate A from Antrodia camphorata induces apoptosis in human liver cancer cells through oxidant-mediated cofilin- and Baxtriggered mitochondrial pathway. Chem Res Toxicol 2010; 23: 125667. 